\title{
The role of the Cincinnati Prehospital Stroke Scale in the emergency department: evidence from a systematic review and meta-analysis
}

This article was published in the following Dove Press journal: Open Access Emergency Medicine

\section{A De Luca ${ }^{1} *$ M Mariani ${ }^{2} *$ MT Riccardi ${ }^{2}$ G Damiani ${ }^{2,3}$}

'Istituti Fisioterapici Ospitalieri, Rome, Italy; ${ }^{2}$ Università Cattolica del Sacro Cuore, Rome, Italy; ${ }^{3}$ Fondazione Policlinico Universitario A. Gemelli Istituto di Ricerca e Cura a Carattere Scientifico (IRCCS), Rome, Italy

*These authors contributed equally to this work
Correspondence: MT Riccardi

Sezione di Igiene, Istituto di Sanità

Pubblica, c/o Istituti Biologici, Largo

Francesco Vito I, Rome 00168, Italy

Tel +390630154396

Email mt.riccardi@gmail.com
Introduction: Stroke is one of the leading causes of morbidity, disability, and mortality in high-income countries. Early prehospital stroke recognition plays a fundamental role, because most clinical decisions should be made within the first hours after onset of symptoms. The Cincinnati Prehospital Stroke Scale (CPSS) is a validated screening tool whose utilization is suggested during triage. The aim of this study is to review the role of the CPSS by assessing its sensitivity and specificity in prehospital and hospital settings.

Methods: A systematic review and a meta-analysis of the literature reporting the CPSS sensitivity and specificity among patients suspected of stroke were undertaken. Electronic databases were searched up to December 2018, and the quality assessment was carried out by using the Revised Quality Assessment of Diagnostic Accuracy Studies -2 (QUADAS-2).

Results: Eleven studies were included in the meta-analysis. Results showed an overall sensitivity of $82.46 \%$ (95\% confidence interval [CI] $74.83-88.09 \%$ ) and specificity of $56.95 \%$ (95\% CI 41.78-70.92). No significant differences were found in terms of sensitivity when CPSS was performed by physicians $(80.11 \%$, 95\% CI $66.14-89.25 \%)$ or non-physicians $(81.11 \%$, 95\% CI 69.78-88.87\%). However, administration by physicians resulted in higher specificity $(73.57 \%$, 95\% CI $65.78-80.12 \%)$ when compared to administration by non-physicians $(50.07 \%$, 95\% CI 31.54-68.58\%). Prospective studies showed higher specificity $71.61 \%$ (95\% CI 61.12-80.18\%) and sensitivity $86.82 \%$ (95\% CI 74.72-93.63) when compared to retrospective studies which showed specificity of $33.37 \%$ (95\% CI 22.79 $45.94 \%$ ) and sensitivity of $78.52 \%$ (95\% CI 75.08-81.60).

Conclusions: The CPSS is a standardized and easy-to-use stroke screening tool whose implementation in emergency systems protocols, along with proper and consistent coordination with local, regional, and state agencies, medical authorities and local experts are suggested.

Keywords: stroke, triage, healthcare, diagnostic accuracy, emergency medical services, emergency department

\section{Introduction}

Nowadays, stroke is the second leading global cause of death after heart disease, and the third leading cause of disability. In 2015, an estimated 6.3 million deaths occurred because of cerebrovascular disease: a total of 3 million people died because of ischemic stroke and 3.3 million because of hemorrhagic stroke. ${ }^{1,2}$ In high-income countries such as Europe, in the last decades, a decreasing trend in stroke mortality rate was reported; for instance, in Italy, from 1990 to 2016, the number of deaths decreased by $17 \%$ (from 60,000 to 50,000 ), and a remarkable decrease by approximately $45 \%$ resulted in Denmark from 1994 to $2011 .^{3-5}$ Despite this declining trend in mortality, stroke 
incidence increased globally by $5 \%$ between 2005 and $2015 .^{3}$ Furthermore in 2010, stroke ranked in the top 18 diseases that contributed to years lived with disability worldwide and, among them, it is the only one that significantly increased from 1990 to $2010 .^{6}$ A significant improvement in patient outcomes is reported by several studies that showed that shorter treatment times increase the chance of returning to good function (ie, being independent and having slight disability or less) when treated within 4.5 hrs from symptoms onset. $^{7-10}$ For this reason, numerous efforts to aid clinicians and Emergency Medical Staff (EMS) to fastly identify this pathology, either in hospital and prehospital settings, were carried out, and several stroke prediction scales were elaborated.

The Cincinnati Prehospital Stroke Scale (CPSS), the Face-Arm-Speech-Time (FAST), the FAST-ED, the Rapid Arterial Occlusion Evaluation Scale, the Los Angeles Prehospital Stroke Screen (LAPSS) are stroke impairment scales developed to quickly assess possible stroke in patients in the prehospital setting. ${ }^{11-15}$ The NIHSS, the Recognition Of Stroke in the Emergency Room, 3-item Stroke Scale, the Cincinnati Prehospital Stroke Severity Scale (CPSSS or C-STAT), were designed for hospital use with the aim of detecting stroke and its severity. ${ }^{16-19}$

In 2013, Jauch et al, reported that the best door-tophysician time should be less than 10 mins, and door-tostroke unit admission time less than 3 hrs. Moreover, EMS are recommended to reach the target time of: less than 20 mins from hospital arrival to CT scan, and less than 60 mins door-to-needle time. ${ }^{20}$ For this reason, emergency medical systems should activate a prehospital stroke prenotification, which is associated both with earlier door-to imaging time (25 mins reduction) and door-to-needle time (60 mins reduction). Currently, the CPSS, the FAST, and the LAPSS scales are recommended by the American Heart Association/American Stroke Association guidelines as validated and standardized tools for stroke screening, even if there is no strong evidence that suggests a higher accuracy of one over the other. ${ }^{21,22}$

The CPSS, proposed by Kothari et al (1999), in particular, is a short, practical, and easy-to-use scale, developed extracting 3 of the 15 symptoms from the NIHSS, the gold standard for the assessment of stroke severity. ${ }^{23,24}$ The CPSS assesses facial palsy, asymmetric arm weakness, and speech disturbances, and each item can be scored as normal or not; if any of three is abnormal, the patient is suspected of having a stroke. ${ }^{11}$
In the last two decades, reviews were published with the aim of comparing existing scales, but none of them focused only on the validity of the CPSS in terms of sensitivity and specificity, even if it is one of the most commonly used prehospital tools, included in several stroke emergency medical systems protocols and national recommendations. ${ }^{25-27}$ The aim of this study is to systematically review the role of the CPSS, globally assessing its sensitivity and specificity in prehospital and hospital settings.

\section{Methods}

\section{Study design and literature search}

A systematic review and meta-analysis of the scientific literature were conducted. Literature search was carried out querying the following electronic databases: EMBASE, PubMed, Web Of Science, Cochrane, and Scopus from their commencements to December 2018, without language restrictions. The search string was created using the elements of the PICO model ( $P$, population/ patient; I, intervention/indicator; $\mathrm{C}$, comparator/control; and O, outcome) and the Preferred Reporting Items for Systematic Reviews and Meta-Analyses check-list and flow diagram were used to collect and report data. ${ }^{28,29}$

The following search terms were used:

1. Terms related to Population: "brain ischemia", "carotid artery diseases", "intracranial embolism and thrombosis", "intracranial hemorrhages", "stroke", "acute cerebrovascular disease", "transient ischemic attack", "cerebrovascular accident", "cerebrovascular diseases", "cerebrovascular disorders", "brain vascular accident", "brain ischemia", "cerebrovascular occlusion";

2. Terms linked to intervention: "Cincinnati Prehospital Stroke Scale";

3. Terms related to measured outcomes: "sensitivity", "specificity", "positive predictive value", "negative predictive value", "reproducibility".

Boolean operators "OR" and "AND" were used to link the keywords.

References of individual studies were also backchecked for relevant studies, and hand search was used to identify missing articles. Two investigators independently screened titles and abstracts of all records to identify potentially relevant publications. 
The following inclusion criteria were used: articles published in English, where the accuracy of the CPSS was assessed using as reference standard the hospital discharge diagnosis of stroke (ischemic, hemorrhagic, or transient ischemic attack).

Articles were excluded if they met at least one of the following criteria: pediatric population, studies without original data (reviews, editorials, practice guidelines, book reviews and chapters, meeting abstracts), quantitative analysis not reported.

Full texts of all potentially eligible studies that met the inclusion criteria were obtained and assessed in duplicate. At all levels, disagreements were resolved by discussion, and by involving a third reviewer when consensus could not be reached.

\section{Quality assessment}

Two independent researchers evaluated the validity of the selected studies using the Revised Quality Assessment of Diagnostic Accuracy Studies -2 (QUADAS-2) tool, a specific validated tool for the quality assessment of diagnostic accuracy studies. ${ }^{30}$

The QUADAS-2 rates the risk of bias in four domains:

1. Patient selection assesses methods of patient selection and inappropriate exclusions;

2. Index test describes how the index test was conducted and interpreted;

3. Reference standard investigates how the reference standard was conducted and interpreted;

4. Flow and timing describes any patients who did not receive the index test(s) and/or reference standard or who were excluded from the TP, TN, FN, FN tables.

The applicability form that follows the first three domains evaluates the correspondence between the study design and the purpose of the specific review to be carried out.

If at least one of the answer in each domain or in the concern regarding applicability was deemed at "high risk of bias", the final risk of bias of the relative domain or in the relative applicability item figures as "High". If the article did not provide sufficient information, the risk of bias figures as "Unclear". Otherwise, if no question found any risk of bias, the domain or the applicability form is scored as "low risk of bias".

Two investigators independently tested the tool for a small number of articles and, once validated, it was used to assess the quality of the included studies.

\section{Data extraction and data analysis}

From each study, data were manually extracted by two authors using a standardized form including the following information: first author's last name, year of publication, country, study design, setting, training in stroke scale of hospital and prehospital staff, administrator of the CPSS, population characteristics, type of stroke evaluated and if CPSS was derived from other source or directly performed. An overall estimation of sensitivity and specificity was achieved using a diagnostic test accuracy meta-analysis of the studies that included data on true positives (TP), true negatives (TN), false positives (FP), and false negatives (FN); when these latter not directly reported, they were derived from available data of the included studies.

Pooled and stratified sensitivity and specificity of CPSS (95\% confidence interval) and summary receiver operating characteristic (sROC) curves were obtained using STATA 13.0 and Cochrane RevMan 5.3. ${ }^{31,32}$ Stratified analyses were performed according to the study design, setting, scale administrator, and type of stroke investigated.

Diagnostic odds ratio (DOR), pooled positive and negative likelihood ratios (LR+ and LR-), were obtained to assess the informative power of the tests.

\section{Results Study selection}

From a total of 448 articles, 386 were excluded after duplicates removal, and title and abstract reading. The remaining 62 articles were selected for full-text review, 44 were excluded because they did not meet the inclusion criteria of this study. A flow diagram describing the article selection process is shown in Figure 1. A total of 18 articles were qualitatively synthesized, and eventually 11 were included in the meta-analysis.

\section{Study and population characteristics}

The included studies were conducted in the following countries: United States, ${ }^{33-41}$ Australia, ${ }^{42,43}$ Italy, ${ }^{44}$ United Kingdom, ${ }^{17}$ Germany, ${ }^{45,46}$ India, ${ }^{47}$ China,${ }^{48}$ and Belgium. ${ }^{49}$ All studies were observational, 6 analyzed a retrospective registry $^{33-35,39-41}$ and 12 a prospective one. ${ }^{17,36-38,42-49}$ Most were conducted in prehospital settings (61\%), ${ }^{33-35,38-43,48,49}$ five in hospital settings, ${ }^{17,37,44-46}$ one both in hospital and prehospital setting, ${ }^{47}$ eventually, another one did not clearly specify the setting. ${ }^{36}$

A total of six studies reported that physicians performed the CPSS scale (neurologists; ${ }^{17,36,45,46}$ emergency 


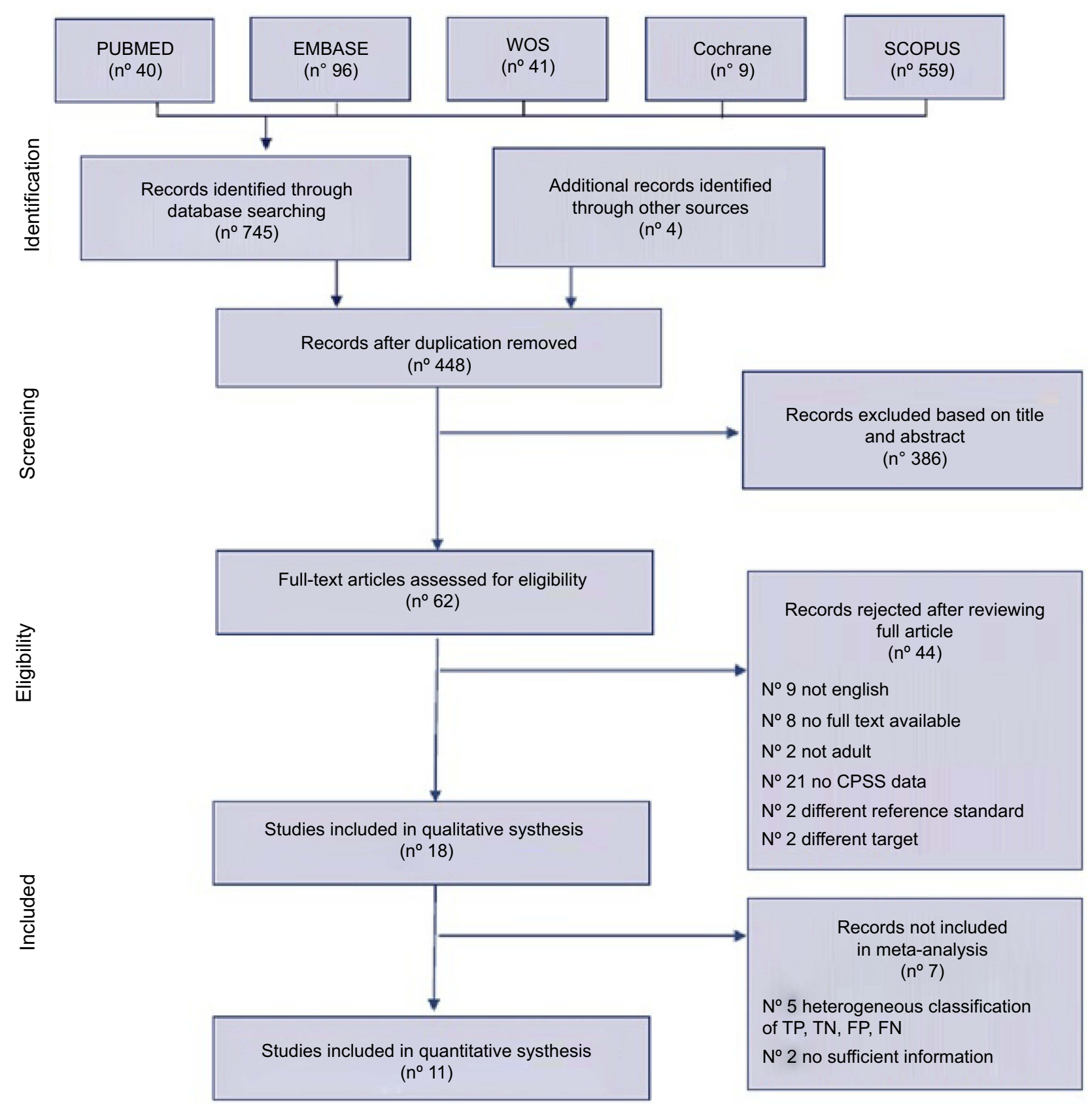

Figure I Flow diagram of included studies.

Abbreviations: WOS, Web of Science; TP, true positives; TN, true negatives; FP, false positives; FN, false negatives.

physicians; ${ }^{48}$ physician certified in the use of the NIHSS ${ }^{37}$ ). In eight studies, the CPSS was derived: from the NIHSS for two studies, ${ }^{36,45}$ from EMS reports for five studies, ${ }^{41-43,46,49}$ and in one from neurological examination in admitted patients. ${ }^{17}$

Almost all studies (72.22\%) investigated all types of stroke (Ischemic, hemorrhagic, and transient ischemic attack); while five studies ${ }^{36,38,40,44,45}$ focused on a particular type of stroke.
The population sizes ranged from 31 to 1,217 , for a total amount of 6,954 subjects enrolled. Mean age ranged from 57.8 to 77 years. Male proportion ranged from $6.93 \%$ to $67.59 \%$. Four studies $(22.22 \%)$ conducted in the US also reported the ethnicity of the population. ${ }^{35,37,40,41}$ In Table 1, a detailed summary of the included studies is reported, including: author, year, country, source, study design, setting of care, administrator, training, sample size, population characteristics, type of 


\begin{tabular}{|c|c|c|c|c|c|c|}
\hline 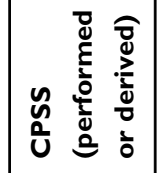 & 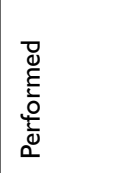 & 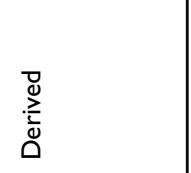 & 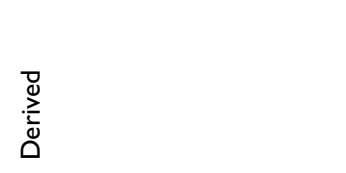 & 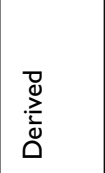 & 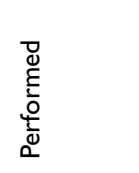 & 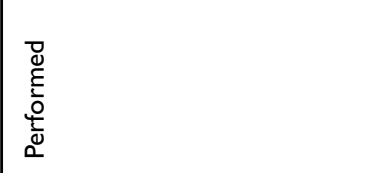 \\
\hline 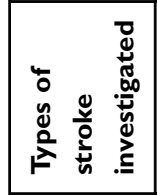 & \begin{tabular}{|l}
$\underline{s}$ \\
$F$ \\
$\underline{\underline{T}}$ \\
$\underline{\underline{x}}$ \\
$\underline{\underline{s}}$
\end{tabular} & $\begin{array}{l}\Delta \\
\vec{r} \\
\underline{\underline{T}} \\
\underline{\underline{v}} \\
\underline{\underline{u}}\end{array}$ & $\begin{array}{l}\overleftrightarrow{s} \\
F \\
\underline{\underline{T}} \\
\underline{\underline{v}} \\
\underline{\underline{s}}\end{array}$ & $\begin{array}{l}\overleftrightarrow{s} \\
F \\
\underline{s} \\
\underline{s} \\
\underline{s}\end{array}$ & $\begin{array}{l}\overleftrightarrow{s} \\
F \\
\underline{\underline{T}} \\
\underline{\underline{s}}\end{array}$ & $\begin{array}{l}\overleftrightarrow{s} \\
t \\
\underline{\underline{T}} \\
\underline{\underline{s}}\end{array}$ \\
\hline 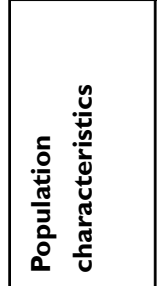 & 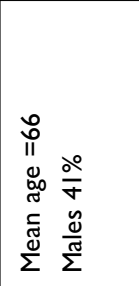 & 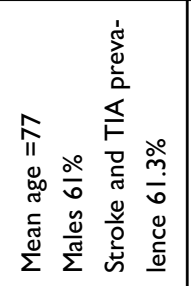 & 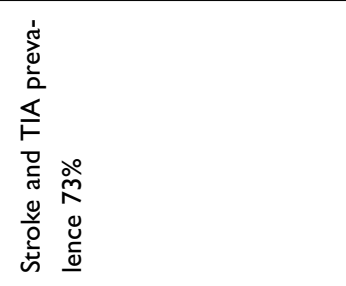 & 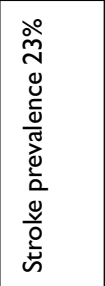 & 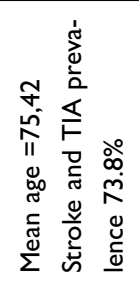 & 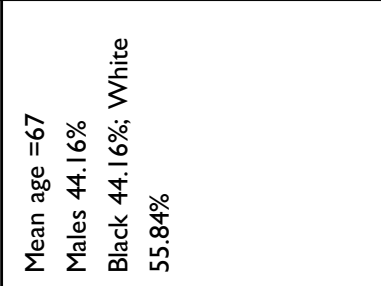 \\
\hline 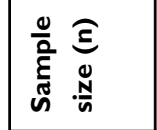 & $\stackrel{\overline{\overline{ }}}{\underline{\underline{y}}}$ & $\bar{m}$ & $\underline{ }$ & 吕 & $\stackrel{\circ}{\underline{m}}$ & $\stackrel{+}{\underline{H}}$ \\
\hline 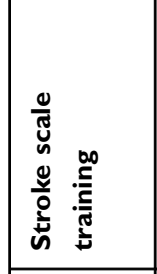 & 1 & 1 & 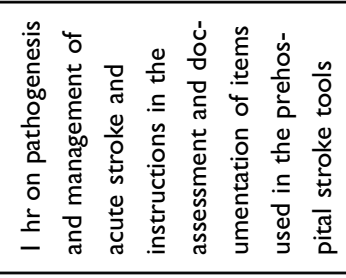 & 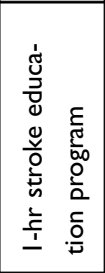 & 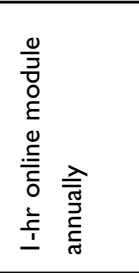 & 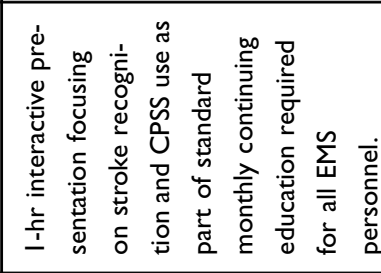 \\
\hline 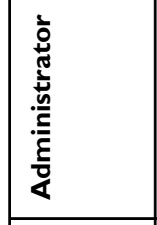 & $\sum_{\Psi}^{n}$ & $\sum_{\Psi}^{n}$ & $\sum_{\Psi}^{n}$ & $\sum_{\tilde{\omega}}^{n}$ & $\sum_{i}^{n}$ & $\sum_{i}^{n}$ \\
\hline 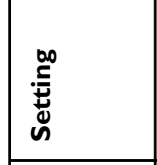 & 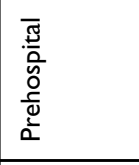 & 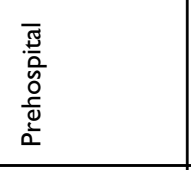 & $\begin{array}{l}\overline{\frac{\pi}{5}} \\
\frac{0}{0} \\
\frac{0}{0} \\
\frac{0}{0}\end{array}$ & 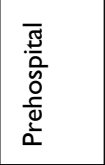 & 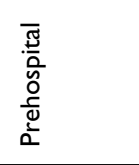 & 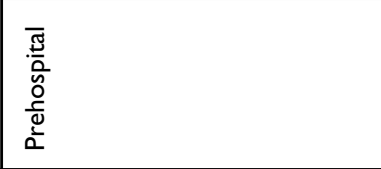 \\
\hline 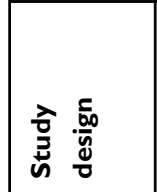 & 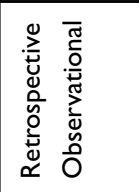 & 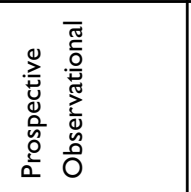 & 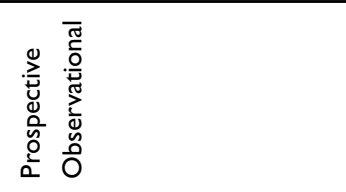 & 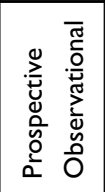 & 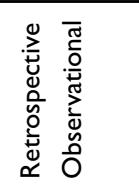 & 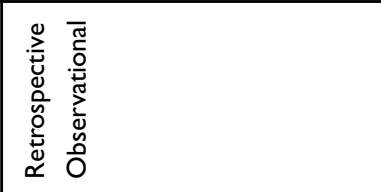 \\
\hline ڤัٌ & 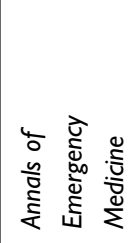 & 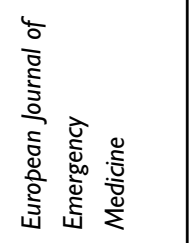 & 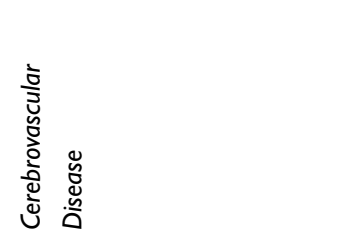 & 总 & 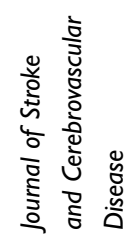 & 善 \\
\hline 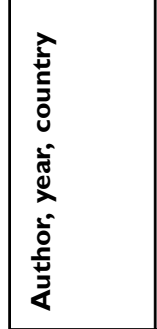 & 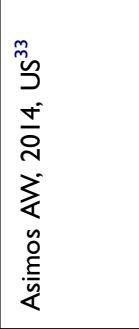 & 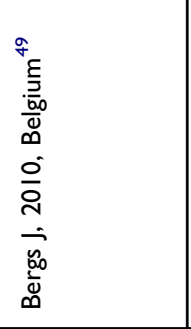 & 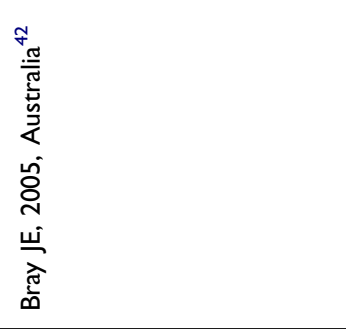 & 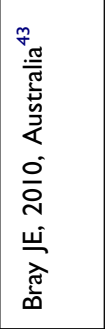 & 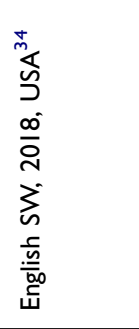 & 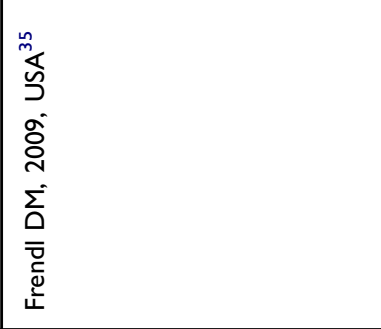 \\
\hline
\end{tabular}




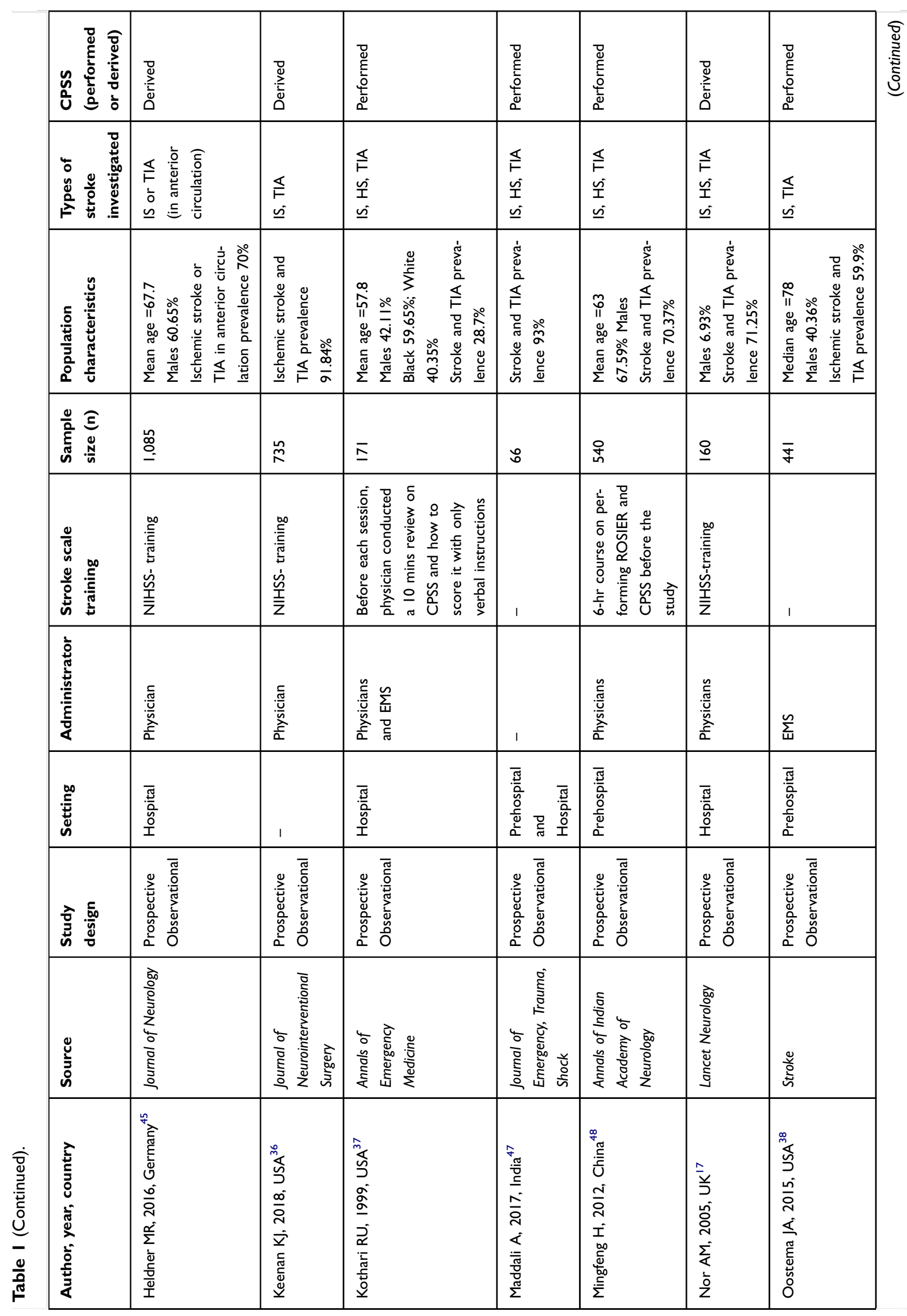




\begin{tabular}{|c|c|c|c|c|c|}
\hline 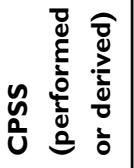 & 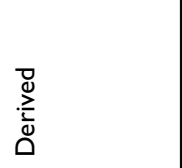 & 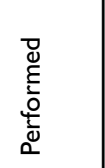 & 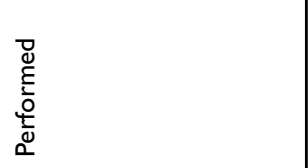 & 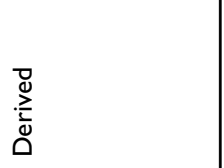 & 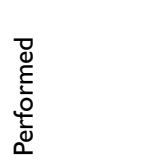 \\
\hline 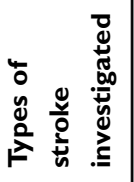 & $\begin{array}{l}\overleftrightarrow{s} \\
\stackrel{s}{I} \\
\underline{v}\end{array}$ & $\begin{array}{l}\overleftrightarrow{s} \\
\stackrel{v i}{I} \\
\underline{v i}\end{array}$ & $\stackrel{\ominus}{\unlhd}$ & $\begin{array}{l}\mathbb{s} \\
\stackrel{v}{\underline{1}} \\
\underline{v}\end{array}$ & $\begin{array}{l}\underline{\underline{I}} \\
\underline{\underline{v}}\end{array}$ \\
\hline 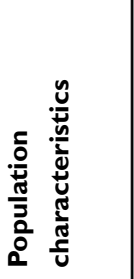 & 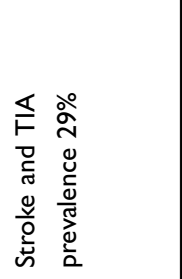 & 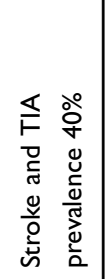 & 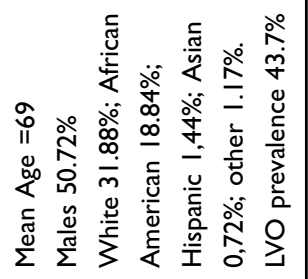 & 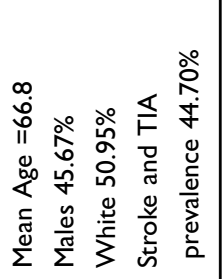 & 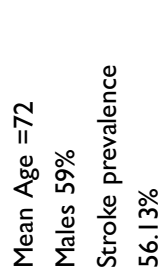 \\
\hline 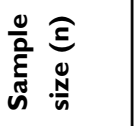 & $\infty$ & 令 & 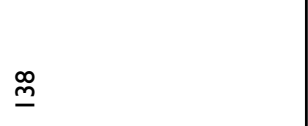 & $\frac{\circ}{\sigma}$ & 虽 \\
\hline 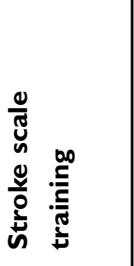 & 1 & 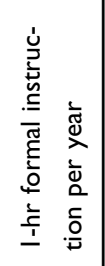 & 1 & 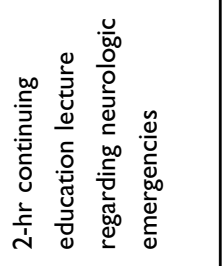 & 1 \\
\hline 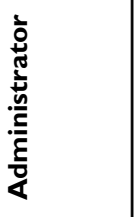 & 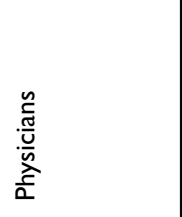 & $\sum_{\Psi}^{\infty}$ & $\sum_{\Psi}^{n}$ & $\sum_{\Psi}^{N}$ & $\sum_{W}^{\infty}$ \\
\hline 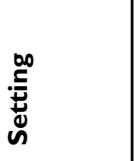 & 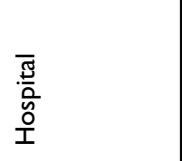 & 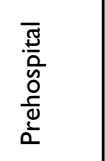 & $\begin{array}{l}\overline{\frac{\pi}{0}} \\
\frac{\overline{0}}{0} \\
\frac{0}{0} \\
\frac{0}{2}\end{array}$ & $\begin{array}{l}\overline{\frac{\pi}{0}} \\
\frac{\overline{0}}{0} \\
\frac{0}{0} \\
\frac{0}{2}\end{array}$ & 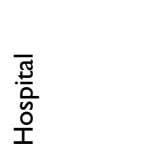 \\
\hline 줄 & 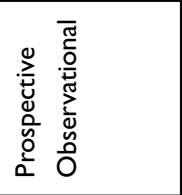 & 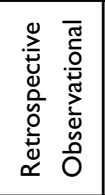 & 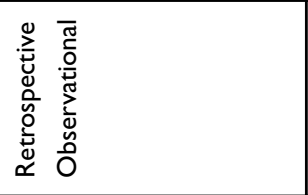 & 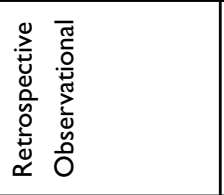 & 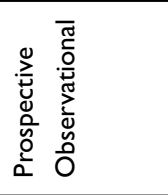 \\
\hline $\begin{array}{l}\stackrel{\Xi}{ } \\
\stackrel{े}{\circ}\end{array}$ & 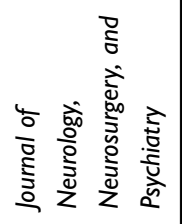 & 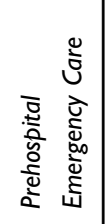 & 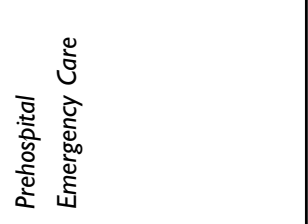 & 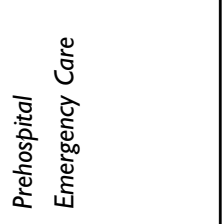 & 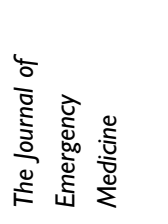 \\
\hline 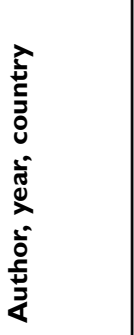 & 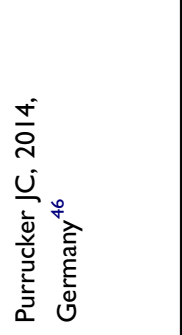 & 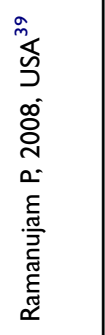 & 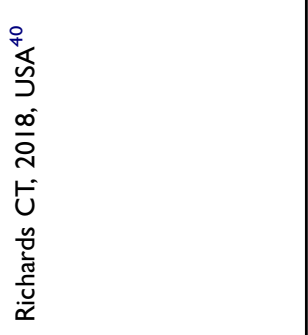 & 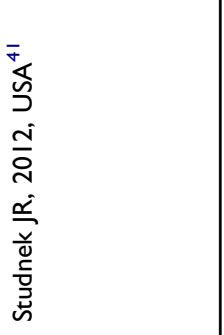 & 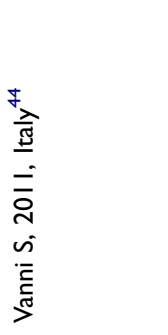 \\
\hline
\end{tabular}


stroke, and whether CPSS was directly performed by the EMS, or derived from other sources.

A total of four studies (22.22\%) specified the characteristics, signs, and symptoms of the population that EMS or physicians identified as eligible to receive CPSS. ${ }^{42,44,48,49}$

\section{Quality assessment}

The overall methodological quality of all included studies $(\mathrm{n}=18)$ is summarized in Table 2 and Figure 2.

No study was deemed at low risk for bias in all the domains, with the exception of one study. ${ }^{48}$

In the first domain that regards the patient selection domain, seven studies ${ }^{33,36-38,44,45,49}$ scored a high risk of bias (sample of patients enrolled in a non-consecutive, nonrandom way or inappropriate exclusions not avoided). In the second domain, index test, only one study ${ }^{36}$ had a high risk of bias, because the CPSS threshold was defined after analysis, and one ${ }^{33}$ scored unclear risk of bias because investigators stated that they did not know whether all of the emergency medical system agencies considered the CPSS as positive when only one of the three symptoms was detected. As to the domain 3, reference standard, five articles reported the blind assessment of the reference standard without knowledge of CPSS results, ${ }^{37,40,44,45,48}$ otherwise, the other studies were reported as having unclear risk of bias because details about the blind assessment were not reported.

Only one study ${ }^{33}$ had an unclear risk of bias in the applicability item: for what concerns the selection of patients, it was not known whether stroke was the reason for transport by EMS or a comorbidity; as to the index test, it is not clear if its interpretation was positive when at least one item was scored as abnormal. Seven studies showed high risks of bias regarding the applicability: five of them included only some types of stroke; $;^{36,38,40,44,45}$ two enrolled admitted patients; $;^{17,37}$ and with regard to the index test, in one study ${ }^{37}$ physicians performed the CPSS but the EMS gave the final score, while in another study ${ }^{17}$ the CPSS results were derived from the neurological exam not performed in emergency setting.

Table 2 Results of the quality assessment according to the Revised Quality Assessment of Diagnostic Accuracy Studies -2 (QUADAS-2) tool

\begin{tabular}{|c|c|c|c|c|c|c|c|}
\hline \multirow[t]{2}{*}{ Study } & \multicolumn{4}{|c|}{ Risk of bias } & \multicolumn{3}{|c|}{ Applicability concerns } \\
\hline & $\begin{array}{c}\text { patient } \\
\text { selection }\end{array}$ & index test & $\begin{array}{l}\text { reference } \\
\text { standard }\end{array}$ & $\begin{array}{c}\text { flow and } \\
\text { timing }\end{array}$ & $\begin{array}{c}\text { patient } \\
\text { selection }\end{array}$ & index test & $\begin{array}{l}\text { reference } \\
\text { standard }\end{array}$ \\
\hline Asimos AW, $2014^{33}$ & $\because$ & ? & $?$ & (:) & ? & ? & (:) \\
\hline Bergs J, $2010^{49}$ & 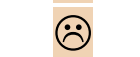 & (:) & ? & (;) & (:) & (:) & (:) \\
\hline Bray JE, $2005^{42}$ & (:) & (:) & ? & (:) & ;: & (:) & (:) \\
\hline Bray JE, $2010^{43}$ & (:) & (:) & ? & (:) & ;: & (:) & (:) \\
\hline English SW, $2018^{34}$ & ;) & ;) & ? & ;) & ;) & ;: & ;) \\
\hline Frendl DM, $2009^{35}$ & (;) & ;) & ? & (:) & ;) & ;) & (:) \\
\hline Heldner MR, $2016^{45}$ & $\because$ & ;) & ;) & ;) & $: 2$ & ;: & ;) \\
\hline Keenan KJ, $2018^{36}$ & 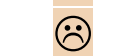 & $\because$ & ? & ;) & $: 2$ & ? & ;: \\
\hline Kothari RU, $1999^{37}$ & 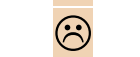 & ;: & (:) & (:) & 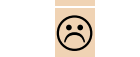 & 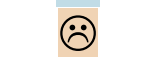 & (:) \\
\hline Maddali A, $2017^{47}$ & (:) & (;) & ? & $\odot$ & (:) & (;) & (:) \\
\hline Mingfeng $\mathrm{H}, 2012^{48}$ & (:) & (:) & (:) & (:) & (:) & (:) & (:) \\
\hline Nor AM, $2005^{17}$ & (:) & (:) & ? & (:) & : & : & (:) \\
\hline Oostema JA, $2015^{38}$ & $\because$ & (:) & ? & (:) & $\because$ & (:) & (:) \\
\hline Purrucker JC, $2014^{46}$ & (:) & ;) & ? & (:) & ;: & (:) & (:) \\
\hline Ramanujam P, $2008^{39}$ & (:) & ;: & ? & ;) & ;) & ;) & ;) \\
\hline Richards CT, $2018^{40}$ & (:) & ;: & ;: & (:) & 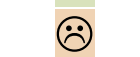 & ;: & ; \\
\hline Studnek CR, $2012^{41}$ & (:) & (:) & ? & (:) & ;: & ;: & (:) \\
\hline Vanni S, $2011^{44}$ & (:) & (:) & (:) & ;: & 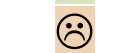 & ;:) & (:) \\
\hline
\end{tabular}

Notes: (;) Low Risk; :) High Risk; ? Unclear Risk. 

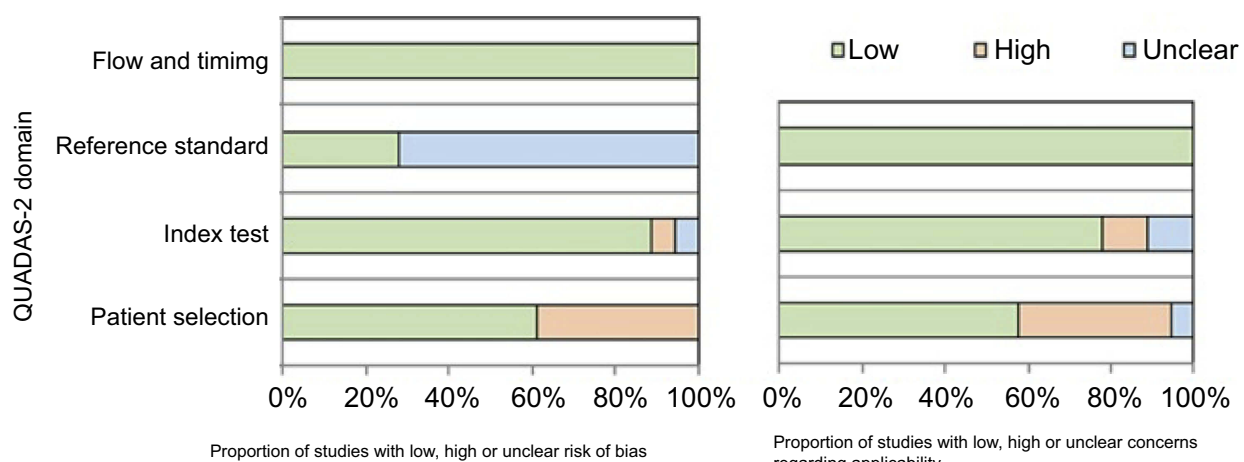

Proportion of studies with low, high or unclear risk of bias

regarding applicability

Figure 2 Stacked bar charts of Revised Quality Assessment of Diagnostic Accuracy Studies -2 (QUADAS-2) scores, presenting a quick overview of the methodological quality of the 18 included studies expressed as a percentage of studies that met each criterion.

\section{CPSS accuracy}

The CPSS TP, TN, FP, and FN, for each study that was included in the meta-analysis, along with their sensitivity and sensibility ( $95 \%$ confidence intervals), and the forest plot are reported in Figure 3.

Among the 11 studies included, the pooled sensitivity and specificity were $82.46 \%$ (95\% CI 74.93-88.09\%) and 56.95 (95\% CI 41.78-70.92\%), respectively, and the sROC is shown Figure 4. The overall DOR was 6.22, the positive likelihood ratio $(\mathrm{LR}+)$ and the negative likelihood ratio (LR-) were 1.92 and 0.31 , respectively.

Data were stratified according to the administrator of the CPSS: physicians or non-physicians.

As for the former, pooled sensitivity was $80.11 \%(95 \% \mathrm{CI}$ $66.14-89.25 \%)$, and pooled specificity was $73.57 \%$ (95\% CI $65.78-80.12 \%$ ). In the latter, pooled sensitivity was $81.11 \%$ (95\% CI 69.78-88.87\%), pooled specificity was 50.07\% (95\% CI 31.54-68.58\%). DOR was 11.21 and 4.30 among physicians and non-physicians, respectively; pooled LR+ and LRwere 3.03 and 0.27 for physicians and 1.62 and 0.38 for nonphysicians, respectively.

$\begin{array}{lrrrrr}\text { Study } & \text { TP } & \text { FP } & \text { FN } & \text { TN } & \text { Sensitivity }(95 \% \text { Cl) } \\ \text { Kothari 1999 } & 117 & 81 & 131 & 531 & 0.47[0.41,0.54] \\ \text { Nor 2005 } & 86 & 12 & 15 & 47 & 0.85[0.77,0.91] \\ \text { Bray 2005 } & 69 & 12 & 4 & 15 & 0.95[0.87,0.98] \\ \text { Frendl 2009 } & 45 & 55 & 16 & 38 & 0.74[0.61,0.84] \\ \text { Bergs 2010 } & 18 & 8 & 1 & 4 & 0.95[0.74,1.00] \\ \text { Bray 2010 } & 176 & 138 & 23 & 513 & 0.88[0.83,0.93] \\ \text { Studnek 2012 } & 147 & 175 & 39 & 55 & 0.79[0.72,0.85] \\ \text { Mingfeng 2012 } & 340 & 49 & 43 & 108 & 0.89[0.85,0.92] \\ \text { Purrucker 2014 } & 145 & 145 & 30 & 320 & 0.83[0.76,0.88] \\ \text { Aslmos 2014 } & 533 & 287 & 130 & 267 & 0.80[0.77,0.83] \\ \text { English 2018 } & 72 & 27 & 24 & 7 & 0.75[0.65,0.83]\end{array}$

Data regarding CPSS accuracy were also stratified according to the study design: pooled sensitivity and specificity among retrospective studies was $78.52 \%$ (95\% CI $75.08-81.60 \%$ ) and $33.37 \%$ (95\% CI $22.79-45.94 \%$ ), respectively (DOR: 1.83; LR+: 1,18; LR-: $0.64 \%$ ); among prospective studies, pooled sensitivity and specificity were $86.82 \%$ (95\% CI $74.72-93.63 \%$ ) and $71.61 \%$ (95\% CI 61.12-80.18\%), respectively (DOR: 16.62; LR+: 3.06; LR-: 0.18).

Data were not sufficient to stratify and assess differences among settings and types of stroke.

Seven studies were not included in meta-analyses. Five studies were not included in the meta-analysis because CPSS TP, TN, FP, FN were not classified in the same way across studies, therefore were not comparable to the classification carried out by the others. ${ }^{36,38,40,44,45}$ The first ${ }^{38}$ excluded hemorrhagic stroke, and did not assess specificity (sensitivity 84.7\%); the second study ${ }^{45}$ evaluated ischemic strokes or TIA of anterior circulation only (sensitivity $72.8 \%$, specificity $70.8 \%$ ); the third ${ }^{36}$ excluded patients with intracranial hemorrhage, incomplete occlusions and occlusions in multiple

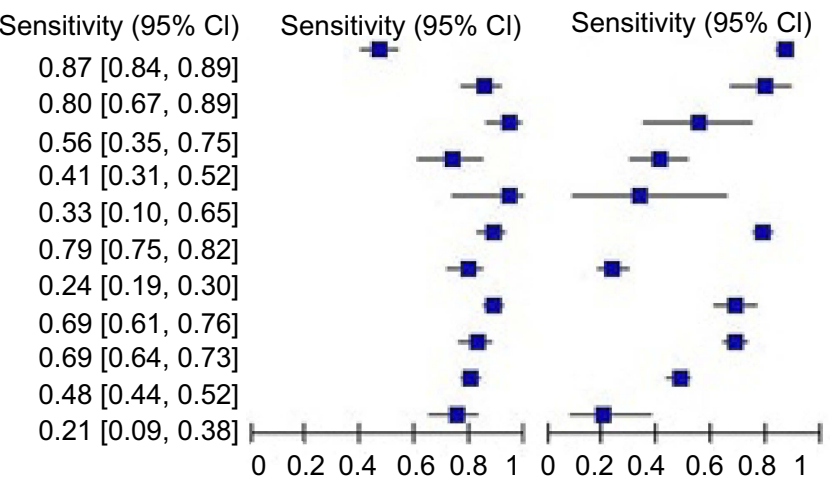

Figure 3 Data from meta-analyzed studies and forest plot for sensitivity and specificity of Cincinnati Prehospital Stroke Scale. Abbreviations: TP, true positives; FP, false positives; FN, false negatives; TN, true negatives. 


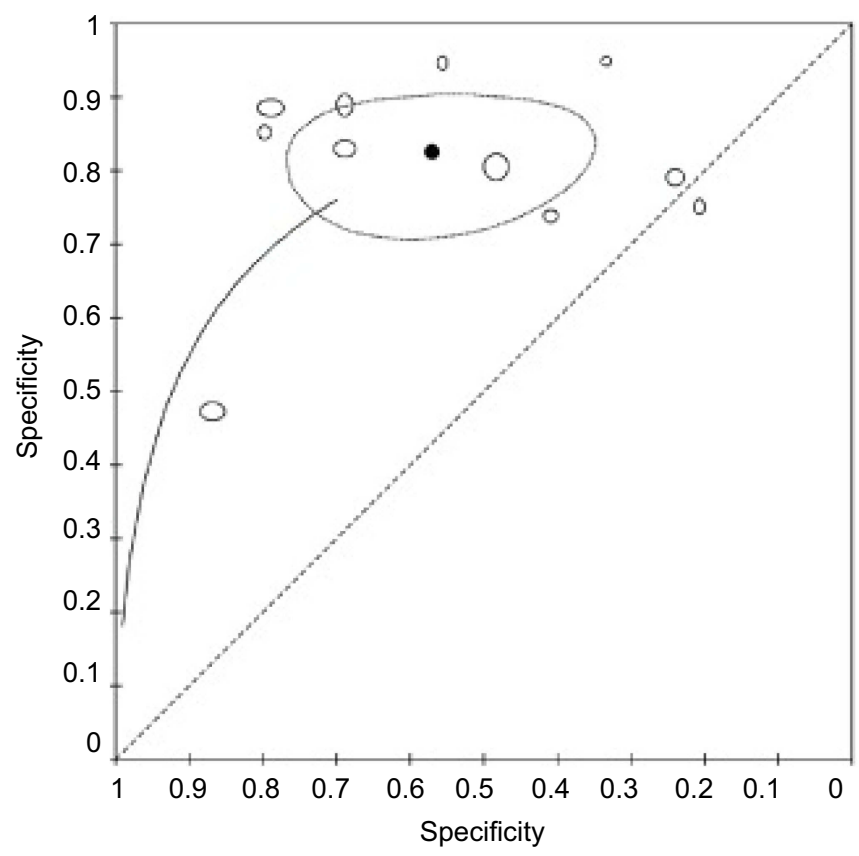

Figure 4 Summary receiving operating characteristic (sROC) curve.

vascular territories (sensitivity 94\%; specificity 29\%); the fourth $^{40}$ included only those with large vessel occlusion (Sensitivity 75\%; specificity $41 \%$ ); eventually, the fifth ${ }^{44}$ assigned TIA patients to non-stroke group, considering them among TN (sensitivity $75 \%$; specificity $78 \%$ ).

Two studies ${ }^{39,47}$ were not included because they did not provide sufficient information about TP, TN, FP, FN. Maddali et $\mathrm{al}^{47}$ included 66 individuals in the study and found that the sensitivity and specificity reached $81.4 \%$ (95\% CI $68.5-$ $90.7 \%$ ) and $100 \%$ (95\% CI $29.2-100 \%$ ), respectively. Furthermore, data were not reported with regard to the CPSS setting of administration (prehospital or hospital setting) and were not stratified according to the type of administrator. Eventually, a study ${ }^{39}$ reported data only regarding the sensitivity $(44 \%$, 95\% CI 39-49\%) but did not assess the specificity.

\section{Discussion}

The results of this systematic review show that the CPSS can be helpful in detecting stroke, with an overall sensitivity of $82.46 \%$ and specificity of $56.95 \%$.

These results are consistent with those of previous systematic reviews and meta-analysis aimed at comparing sensitivity and specificity of other scales. ${ }^{50-52}$ In fact, the CPSS has shown similar outcomes in terms of sensitivity and specificity when compared to other scales that are also recommended in prehospital setting, ${ }^{21}$ such as the FAST scale, which investigates the same areas of the CPSS but without detailed questions to be addressed; and the LAPSS, which assesses motor asymmetries along with blood glucose level and anamnestic features. In contrast to the CPSS, the FAST scale evaluates the speech item by using the entire conversation with the individual suspected of stroke, without using a specific sentence to be repeated; for this reason the CPSS might have the advantage to be more reproducible and more standardized among providers; ${ }^{46}$ the LAPSS takes about 5 mins to be performed, while the CPSS requires about 1.5 mins. ${ }^{53,54}$

However, it should be considered that a positive CPSS does not necessarily result in a stroke diagnosis. This may be appreciated by looking at LR + and LR-, which provide an idea of the test utility. As a matter of fact, LR+ has a small value in every examined scenario, so an abnormal CPSS result is more frequent in stroke patients than in healthy ones but not significantly. On the other hand, the likelihood ratio for a negative result (LR-) showed good values ( 0.27 and 0.18$)$ in the two best scenarios (when performed by physicians and in prospective studies, respectively). ${ }^{55}$ This means that CPSS can be considered a good triage tool when performed in the best way because the odds of having a stroke considerably decrease when the test is negative.

Some sources of heterogeneity were investigated with regard to CPSS accuracy, others were hypothesized, but data were not available for assessing, for instance, differences among types of stroke or among settings. 
Most studies (77.78\%) did not report the eligibility criteria for which the scale had to be administered to the study population. This might lead to an increase in heterogeneity and reduction in terms of the comparability among the study populations. Prospective studies showed a higher accuracy above all in terms of specificity (increase of specificity of almost $40 \%$, from $33.37 \%$ in retrospective studies to $71.60 \%$ in prospective ones) and of sensitivity (increase of sensitivity of $8 \%$, from $78.52 \%$ to $86.82 \%$ ). A recent systematic review, that analyzed several prehospital scales (not including CPSS), reported that looking at the forest plot distribution according to study design stroke screening scales performed better in retrospective studies when compared to prospective ones, and this may be due to a "difficult application of some scales in a real 'on-air' situation". ${ }^{56}$ In this review, that focuses on the CPSS, a better accuracy in prospective studies was found, probably because the previous review ${ }^{56}$ analyzed more complex prehospital scales. In fact, the CPSS is a very simple scale, easily applicable in the emergency medical system. For this reason, when planning to start a prospective study on the CPSS, an educational intervention that underlines the importance both of a correct administration of the tool and data collection, might be more effective. Another source of heterogeneity was identified among the administrators of the CPSS. When physicians and non-physicians were compared sensitivity showed similar results $(80.11 \%$ for the former and $81.11 \%$ for the latter group), while specificity increased by $46.94 \%$, from $50.07 \%$ - among non-physicians - to $73.57 \%$ - among physicians. This might be due to the level of expertise achieved with education and training in the detection of signs of stroke during the performance of the CPSS; eg, one of the most recurrent mistakes while assessing the CPSS items is to simply ask patients to "smile" rather than "smile, showing your teeth", ${ }^{52}$ weakening the accuracy of this scale.

For this reason, the educational component may need further evaluation, and it is suggested that for future studies the educational intervention they implemented should be reported.

A multilevel educational stroke program could be planned in order to reduce misclassification errors (FN or FP), also with the aim of lowering overtriage and undertriage. The CPSS, as a triage test, should help to maximize the number of patients with suspected stroke who need access to hospitals as soon as possible, because more subsequent test is performed to exclude FP. ${ }^{57,58}$

Since a low specificity parallels overtriage, it is suggested to locally calculate a threshold to define an overtriage as acceptable or not, because it depends on the capacity of the system to treat patients with suspected stroke in addition to the baseline caseload. ${ }^{59,60}$

Results from this review take into account the outcome that CPSS achieves in predicting stroke without distinguishing between ischemic, hemorrhagic stroke, and TIA. This could be important from the organizational perspective since all of the three pathologies share the same destination, ie, an advanced care center. It has been proven, in fact, that hemorrhagic strokes need a specialized evaluation to undergo supportive care or neurosurgery, and TIAs need monitoring because of the added risk of ischemic stroke, hospitalization for cardiovascular events, and death. ${ }^{2,61}$

\section{Strengths and limitations of the study}

The strengths of this review include a robust systematic process for search strategy, appraisal, data extraction, and description, supplemented with hand searching and forward citation searching. All screening and data extraction processes were performed by two independent reviewers. A limitation concerns the overall low quality of most of the included studies; however, as previously reported the risk of bias when examining diagnostic tools is generally high. ${ }^{62,63}$

A strength lies in the fact that CPSS is evaluated in real-world conditions, indirectly providing important information on its repeatability, reproducibility (standardized instruction to detect the signs), and ease of use. ${ }^{64}$

Further research is needed to cover a wider perspective that takes into account the evaluation of a system by also addressing the additional value of patient safety, the ethical and socio-cultural implications, the organizational impact, and a cost-effectiveness analysis of the CPSS. This may help to understand and to quantify the global impact that might be brought by the introduction of this scale.

\section{Conclusion}

In conclusion, this research helps to explain and give an updated vision of the value of the CPSS as a screening tool that is aimed at supporting the diagnosis of stroke. It showed to be a simple, reproducible, easy to teach and to use the tool. For this reason, the implementation of emergency systems protocols, especially in hospital triage, that take into account the use of validated and standardized instrument for stroke screening as the CPSS, along with a proper and consistent evaluation and coordination among local, regional, state agencies, medical authorities and local experts, should be undertaken. 


\section{Disclosure}

The authors report no conflicts of interest in this work.

\section{References}

1. Setacci C, Lanza G, Ricci S, et al.; Stroke Prevention and Educational Awareness Diffusion (SPREAD). SPREAD Italian Guidelines for stroke. Indications for carotid endarterectomy and stenting. J Cardiovasc Surg (Torino). 2009;50(2):171-182.

2. Benjamin EJ, Virani SS, Callaway CW, et al. Heart disease and stroke statistics $3 / 42018$ update: a report from the American Heart Association. Circulation. 2018. doi:10.1161/CIR.0000000000000558

3. GBD 2016 Disease and Injury Incidence and Prevalence Collaborators. Global, regional, and national incidence, prevalence, and years lived with disability for 328 diseases and injuries for 195 countries, 1990-2016: a systematic analysis for the Global Burden of Disease Study 2016. Lancet. 2017;390(10100):1211-1259.

4. Schmidt M, Jacobsen JB, Johnsen SP, Bøtker HE, Sørensen HT. Eighteenyear trends in stroke mortality and the prognostic influence of comorbidity. Neurology. 2014;82(4):340-350. doi:10.1212/WNL.0000000000000062

5. Koton S, Schneider ALC, Rosamond WD, et al. Stroke incidence and mortality trends in US communities, 1987 to 2011. JAMA. 2014;312 (3):259-268. doi:10.1001/jama.2014.7692

6. US Burden of Disease Collaborators. The state of US health, 19902010: burden of diseases, injuries, and risk factors. JAMA. 2013;310:591-608. doi:10.1001/jama.2013.13805

7. Saver JL. Time is brain-quantified. Stroke. 2006;37(1):263-266. doi:10.1161/01.STR.0000196957.55928.ab

8. Lees KR, Bluhmki E, von Kummer R, et al. Time to treatment with intravenous alteplase and outcome in stroke: an updated pooled analysis of ECASS, ATLANTIS, NINDS, and EPITHET trials. Lancet. 2010;375(9727):1695-1703. doi:10.1016/S0140-6736(10)60491-6

9. Pashapour A, Atalu A, Farhoudi M, et al. Early and intermediate prognosis of intravenous thrombolytic therapy in acute ischemic stroke subtypes according to the causative classification of stroke system. Pak J Med Sci. 2013;29(1):181-186. doi:10.12669/pjms.291.2897

10. Wardlaw JM, Murray V, Berge E, et al. Recombinant tissue plasminogen activator for acute ischaemic stroke: an updated systematic review and meta-analysis. Lancet. 2012;379(9834):2364-2372. doi:10.1016/S0140-6736(12)60738-7

11. Kothari RU, Hall K, Brott T, Broderick J. Early stroke recognition: developing an out-of-hospital NIH Stroke Scale. Acad Emerg Med. 1997;4(10):986-990.

12. Harbison J, Hossain O, Jenkinson D, Davis J, Louw SJ, Ford GA. Diagnostic accuracy of stroke referrals from primary care, emergency room physicians, and ambulance staff using the face arm speech test. Stroke. 2003;34:71-76. doi:10.1161/01.STR.0000044170.46643.5E

13. Lima FO, Furie KL, Frankel MH, et al. The field assessment stroke triage for emergency destination (FAST-ED): a simple and accurate pre-hospital scale to detect large vessel occlusion strokes. Stroke. 2016;47(8):19972002.

14. De la Ossa NP, Carrera D, Gorchs M, et al. Design and validation of a prehospital stroke scale to predict large arterial occlusion: the rapid arterial occlusion evaluation scale. Stroke. 2014;45(1):87-91. doi:10.1161/STROKEAHA.113.003071

15. Kidwell CS, Saver JL, Schubert GB, Eckstein M, Starkman S. Design and retrospective analysis of the Los Angeles Prehospital Stroke Screen (LAPSS). Prehosp Emerg Care. 1998;2(4):267-273.

16. Spilker J, Kongable G, Barch C, et al. Using the NIH Stroke Scale to assess stroke patients. J Neurosci Nurs. 1997;29(6):384-393.

17. Nor AM, Davis J, Sen B, et al. The Recognition of Stroke in the Emergency Room (ROSIER) scale: development and validation of a stroke recognition instrument. Lancet Neurol. 2005;4(11):727-734. doi:10.1016/S1474-4422(05)70201-5
18. Singer OC, Dvorak F, de Rochemont R, Lanfermann H, Sitzer M, Neumann-Haefelin T. A simple 3-item stroke scale: comparison with the National Institutes of Health Stroke Scale and prediction of middle cerebral artery occlusion. Stroke. 2005;36(4):773-776. doi:10.1161/01.STR.0000157591.61322.df

19. Katz BS, McMullan JT, Sucharew H, Adeoye O, Broderick JP. Design and validation of a prehospital scale to predict stroke severity: cincinnati prehospital stroke severity scale. Stroke. 2015;46(6):15081512. doi:10.1161/STROKEAHA. 115.008804

20. Jauch E, Saver J, Adams H, et al. Guidelines for the early management of patients with acute ischemic stroke: a guideline for healthcare professionals from the American Heart Association/American Stoke Association. Stroke. 2013;44:870-947. doi:10.1161/ STR.0b013e318284056a

21. Powers WJ, Rabinstein AA, Ackerson T, et al. 2018 guidelines for the early management of patients with acute ischemic stroke: a guideline for healthcare professionals from the American Heart Association/American Stroke Association. Stroke. 2018;49:e233e234. doi:10.1161/STR.0000000000000158

22. Keenan KJ, Kircher C, McMullan JT. Prehospital prediction of large vessel occlusion in suspected stroke patients. Curr Atheroscler Rep. 2018;20(7):34. doi:10.1007/s11883-018-0734-x

23. Heldner MR, Zubler C, Mattle HP, et al. National Institutes of Health stroke scale score and vessel occlusion in 2152 patients with acute ischemic stroke. Stroke. 2013;44(4):1153-1157. doi:10.1161/ STROKEAHA.111.000604

24. Fischer U, Arnold M, Nedeltchev K, et al. NIHSS score and arteriographic findings in acute ischemic stroke. Stroke. 2005;36(10):21212125. doi:10.1161/01.STR.0000182099.04994.fc

25. Glober NK, Sporer KA, Guluma KZ, et al. Acute stroke: current evidence-based recommendations for prehospital care. West J Emerg Med. 2016;17(2):104. doi:10.5811/westjem.2015.12.28995

26. Mazzucco S, Turri G, Mirandola R, Bovi P, Bisoffi G, Verona Hospital Stroke Study Group. What is still missing in acute-phase treatment of stroke: a prospective observational study. Neurol Sci. 2013;34(4):449-455. doi:10.1007/s10072-012-1024-y

27. CConsoli D, De Falco FA, Lenzi GL, et al. Organizzazione dell'assistenza all'ictus: le Stroke Unit. [Stroke Care Organization: Stroke Units]. Rome: Quaderni del Ministero della Saulte [Ministry of Health Acts]; 2010;2:23. Italian.

28. Richardson WS, Wilson MC, Nishikawa J, Hayward RS. The well-built clinical question: a key to evidence-based decisions. ACP J Club. 1995;123:A12-3.

29. Moher D, Liberati A, Tetzlaff J, Altman DG, PRISMA Group. Preferred reporting items for systematic reviews and meta-analyses: the PRISMA statement. Ann Intern Med. 2009;151(4):264-269, W64. doi:10.7326/0003-4819-151-4-200908180-00135

30. Whiting PF, Rutjes AW, Westwood ME, et al. QUADAS-2: a revised tool for the quality assessment of diagnostic accuracy studies. Ann Intern Med. 2011;155(8):529-536. doi:10.7326/0003-4819-155-8201110180-00009

31. StataCorp. Stata Statistical Software: Release 13. College Station (TX): StataCorp LP; 2013.

32. Review manager (RevMan) [Computer program]. Version 5.3. Copenhagen: The Nordic Cochrane Centre, The Cochrane Collaboration, 2014.

33. Asimos AW, Ward S, Brice JH, Rosamond WD, Goldstein LB, Studnek J. Out-of-hospital stroke screen accuracy in a state with an emergency medical services protocol for routing patients to acute stroke centers. Ann Emerg Med. 2014;64(5):509-515. doi:10.1016/j. annemergmed.2014.03.024

34. English SW, Rabinstein AA, Mandrekar J, Klaas JP. Rethinking prehospital stroke notification: assessing utility of emergency medical services impression and cincinnati prehospital stroke scale. $J$ Stroke Cerebrovasc Dis. 2018;27(4):919-925. doi:10.1016/j. jstrokecerebrovasdis.2017.10.036 
35. Frendl DM, Strauss DG, Underhill BK, Goldstein LB. Lack of impact of paramedic training and use of the cincinnati prehospital stroke scale on stroke patient identification and on-scene time. Stroke. 2009;40(3):754 756. doi:10.1161/STROKEAHA.108.531285

36. Keenan KJ, Smith WS. The Speech Arm Vision Eyes (SAVE) scale predicts large vessel occlusion stroke as well as more complicated scales. J Neurointerv Surg.

37. Kothari RU, Pancioli A, Liu T, Brott T, Broderick J. Cincinnati prehospital stroke scale: reproducibility and validity. Ann Emerg Med. 1999;33(4):373-378.

38. Oostema JA, Konen J, Chassee T, Nasiri M, Reeves MJ. Clinical predictors of accurate prehospital stroke recognition. Stroke. 2015;46 (6):1513-1517. doi:10.1161/STROKEAHA.115.008650

39. Ramanujam P, Guluma KZ, Castillo EM, et al. Accuracy of stroke recognition by emergency medical dispatchers andparamedics - San Diego experience. Prehosp Emerg Care. 2008;12(3):307-313. doi:10.1080/10903120802099526

40. Richards CT, Huebinger R, Tataris KL, et al. Cincinnati prehospital stroke scale can identify large vessel occlusion stroke. Prehosp Emerg Care. 2018;22(3):312-318. doi:10.1080/10903127.2017.1387629

41. Studnek JR, Asimos A, Dodds J, Swanson D. Assessing the validity of the Cincinnati prehospital stroke scale and the medic prehospital assessment for code stroke in an urban emergency medical services agency. Prehosp Emerg Care. 2013;17(3):348-353. doi:10.3109/ 10903127.2013.773113

42. Bray JE, Martin J, Cooper G, Barger B, Bernard S, Bladin C. Paramedic identification of stroke: community validation of the Melbourne ambulance stroke screen. Cerebrovasc Dis. 2005;20 (1):28-33. doi:10.1159/000086201

43. Bray JE, Coughlan K, Barger B, Bladin C. Paramedic diagnosis of stroke: examining long-term use of the Melbourne Ambulance Stroke Screen (MASS) in the field. Stroke. 2010;41(7):1363-1366. doi:10.1161/STROKEAHA.109.571836

44. Vanni S, Polidori G, Pepe G, et al. Use of biomarkers in triage of patients with suspected stroke. J Emerg Med. 2011;40(5):499-505. doi:10.1016/j.jemermed.2008.09.028

45. Heldner MR, Hsieh K, Broeg-Morvay A, et al. Clinical prediction of large vessel occlusion in anterior circulation stroke: mission impossible? J Neurol. 2016;263(8):1633-1640. doi:10.1007/s00415-016-8180-6

46. Purrucker JC, Hametner C, Engelbrecht A, Bruckner T. Comparison of stroke recognition and stroke severity scores for stroke detection in a single cohort. J Neurol Neurosurg Psychiatry. 2015;86(9):10211028. doi:10.1136/jnnp-2014-309260

47. Maddali A, Razack FA, Cattamanchi S, Ramakrishnan TV. Validation of the cincinnati prehospital stroke scale. J Emerg Trauma Shock. 2018;11(2):111. doi:10.4103/JETS.JETS_8_17

48. Mingfeng H, Zhixin W, Qihong G, Lianda L, Yanbin Y, Jinfang F. Validation of the use of the ROSIER scale in prehospital assessment of stroke. Ann Indian Acad Neurol. 2012;15(3):191. doi:10.4103/ 0972-2327.99713

49. Bergs J, Sabbe M, Moons P. Prehospital stroke scales in a Belgian prehospital setting: a pilot study. Eur J Emerg Med. 2010;17(1):2-6. doi:10.1097/MEJ.0b013e32831040ec
50. Rudd M, Buck D, Ford GA, Price CI. A systematic review of stroke recognition instruments in hospital and prehospital settings. Emerg Med J. 2016;33(11):818-822. doi:10.1136/emermed-2015205197

51. Brandler ES, Sharma M, Sinert RH, Levine SR. Prehospital stroke scales in urban environments: a systematic review. Neurology. 2014;82(24):2241-2249. doi:10.1212/WNL.0000000000000523

52. Zhelev Z, Walker G, Henschke N, Fridhandler J, Yip S. Prehospital stroke scales as screening tools for early identification of stroke and transient ischemic attack. Cochrane Database Syst Rev. 2019;4: CD011427.

53. Hurwitz AS, Brice JH, Overby BA, Evenson KR. Directed use of the cincinnati prehospital stroke scale by laypersons. Prehosp Emerg Care. 2005;9(3):292-296. doi:10.1080/10903120590962283

54. Chen S, Sun H, Lei Y, et al. Validation of the Los Angeles prehospital stroke screen (LAPSS) in a Chinese urban emergency medical service population. PLoS One. 2013;8(8):e70742. doi:10.1371/ journal.pone.0070742

55. Riewe E, Neubauer E, Pfeifer AC, Schiltenwolf M. Predicting persistent back symptoms by psychosocial risk factors: validity criteria for the ÖMPSQ and the HKF-R 10 in Germany. PLoS One. 2016;11(7):e0158850. doi:10.1371/journal.pone.0158850

56. Vidale S, Agostoni E. Prehospital stroke scales and large vessel occlusion: a systematic review. Acta Neurol Scand. 2018;138(1):2431. doi:10.1111/ane. 12908

57. Leeflang M. Systematic reviews and meta-analyses of diagnostic test accuracy. Clin Microbiol Infect. 2014;20(2):105-113. doi:10.1111/ 1469-0691.12474

58. Pride GL, Fraser JF, Gupta R, et al. Prehospital care delivery and triage of stroke with emergent large vessel occlusion (ELVO): report of the Standards and Guidelines Committee of the Society of Neurointerventional Surgery. J Neurointerv Surg. 2017;9(8):802812. doi:10.1136/neurintsurg-2016-012699

59. Bogle BM, Asimos AW, Rosamond WD. Regional evaluation of the severity-based stroke triage algorithm for emergency medical services using discrete event simulation. Stroke. 2017;48(10):28272835. doi:10.1161/STROKEAHA.117.017905

60. Dozois A, Hampton L, Kingston CW, et al. PLUMBER Study (prevalence of large vessel occlusion strokes in Mecklenburg county emergency response). Stroke. 2017;48(12):3397-3399. doi:10.1161/ STROKEAHA.117.018925

61. Van Gaal S, Demchuk A. Clinical and technological approaches to the prehospital diagnosis of large vessel occlusion. Stroke. 2018;49 (4):1036-1043. doi:10.1161/STROKEAHA.117.017947

62. Biondi-Zoccai G, editor. Diagnostic Meta-Analysis: A Useful Tool for Clinical Decision-Making. Berlin: Springer International Publishing; 2018.

63. Li L, Tian J, Tian H, Sun R, Liu Y, Yang K. Quality and transparency of overviews of systematic reviews. J Evid Based Med. 2012;5 (3):166-173. doi:10.1111/j.1756-5391.2012.01185.x

64. Kosack CS, Page AL, Klatser PR. A guide to aid the selection of diagnostic tests. Bull World Health Organ. 2017;95(9):639. doi:10.2471/BLT.16.187468

\section{Publish your work in this journal}

The Open Access Emergency Medicine is an international, peerreviewed, open access journal publishing original research, reports, editorials, reviews and commentaries on all aspects of emergency medicine. The manuscript management system is completely online and includes a very quick and fair peer-review system, which is all easy to use. Visit http://www.dovepress.com/testimonials.php to read real quotes from published authors. 